University of Wollongong

Research Online

Faculty of Informatics - Papers (Archive)

Faculty of Engineering and Information

Sciences

$1-1-2008$

\title{
Correlations of Length Distributions between Non-coding and Coding Sequences of Arabidopsis Thaliana
}

\author{
Rachel Caldwell \\ University of Wollongong, rac34@uowmail.edu.au \\ Yan-Xia Lin \\ University of Wollongong, yanxia@uow.edu.au \\ Ren Zhang \\ University of Wollongong, rzhang@uow.edu.au
}

Follow this and additional works at: https://ro.uow.edu.au/infopapers

Part of the Physical Sciences and Mathematics Commons

\section{Recommended Citation}

Caldwell, Rachel; Lin, Yan-Xia; and Zhang, Ren: Correlations of Length Distributions between Non-coding and Coding Sequences of Arabidopsis Thaliana 2008, 72-77.

https://ro.uow.edu.au/infopapers/1409

Research Online is the open access institutional repository for the University of Wollongong. For further information contact the UOW Library: research-pubs@uow.edu.au 


\title{
Correlations of Length Distributions between Non-coding and Coding Sequences of Arabidopsis Thaliana
}

\begin{abstract}
Gene length and organization are important attributes of genomics. With a large amount of sequence data becoming available, statistical analyses can be applied to this data and will offer beneficial output to research communities. Previous work in this field has focused on protein length and its coding region, while we are also investigating the non-coding regions, as well as trying to uncover any potential correlation that may exist between the regions. Analysis on the Arabidopsis thaliana found there was a strong correlation between the coding sequence length and the 3 UTR region, conditional on the 5 UTR ratios. These results seemed consistent over all chromosomes and data that either contained or lacked introns. Classification of proteins into functional classes presented several interesting results. It was found that the number of proteins in a specific functional category decreased as the value of the 5 UTR ratio length, separated into eight subsets, increased. This work has revealed some possible correlations between different gene regions.
\end{abstract}

\section{Keywords}

Correlations, Length, Distributions, between, Non, coding, Coding, Sequences, Arabidopsis, Thaliana

\section{Disciplines}

Physical Sciences and Mathematics

\section{Publication Details}

Caldwell, R., Lin, Y. \& Zhang, R. (2008). Correlations of Length Distributions between Non-coding and Coding Sequences of Arabidopsis Thaliana. IEEE International Conference on Bioinformatics and Biomedicine, BIBM 2008 (pp. 72-77). Los Alamitos, California, Washington, Tokyo: IEEE Computer Society. 


\title{
Correlations of length distributions between non-coding and coding sequences of Arabidopsis thaliana
}

\author{
Rachel Caldwell, Yan-Xia Lin, Ren Zhang \\ School of Biological Science, School of Mathematics and Applied Statistics \\ University of Wollongong, Australia \\ rac34@uow.edu.au
}

\begin{abstract}
Gene length and organization are important attributes of genomics. With a large amount of sequence data becoming available, statistical analyses can be applied to this data and will offer beneficial output to research communities. Previous work in this field has focused on protein length and its coding region, while we are investigating the noncoding and coding regions, as well as trying to uncover any potential correlation that may exist between these regions. Analysis on the Arabidopsis thaliana found there was a strong correlation between the coding sequence length and non-coding regions, conditional on the 5' UTR data. These results seemed consistent over all chromosomes and data that either contained or lacked introns. Classification of proteins into functional classes presented several interesting results. It was found that the number of protein types in a specific functional category decreased as the value of the 5, UTR ratio, separated into eight subsets, increased. This work has revealed some possible correlations between different gene regions.
\end{abstract}

\section{Introduction}

With numerous DNA sequences now publicly available to researchers, much more information can be extracted and interpreted. Therefore, there is a potential to gain invaluable biological information from these sequences. While much attention within computational biology research has focused on identifying gene products and locations from experimentally obtained DNA sequences, several studies have given attention to protein length variations amid the three domains of life. It was found that there was a noticeable increase in protein length observed in eukaryotes compared to prokaryotes $[1,2]$. Other studies established there was a positive correlation between average protein lengths and genome complexity [3].

Less is known of the length distributions of noncoding regions. One study evaluated the distances between neighboring genes and the lengths of the 3' un-translated regions (UTRs) and it has been found that length and distance between genes and their corresponding un-translated regions had important implications in gene expression [4].

Previous studies of our group have showed that the transcription start site to the translation start site distance distribution varies among different organisms and each organism has it own specific character [5]. This is consistent with aforementioned studies focusing on protein length, with similar results in the increase in distance from simple prokaryotes to more complicated eukaryotic organisms.

The sequencing project of Arabidopsis thaliana, a popular model organism due to its rapid life cycle, and relatively small genome is benefiting the wider plant science community with the information gained from the sequencing data, and contributing to a generalized view on plant genes. It may also offer insight into the differences between higher plants and other major groupings of living organisms [6].

With the full-length cDNA technology coming of age, and now readily available, this data is not only useful with intron and exon studies, but the untranslated messenger RNA regions which are important in understanding transcription initiation and termination processes. A recent study conducted using the Arabidopsis cDNA data discovered many features of gene structure and organization [7]. The 5' and 3' UTR data for the large dataset confirmed previous study results, suggesting the average length of the 5' UTR length ranges between 100 to 200 nucleotides, whereas the 3 ' UTRs are much more variable [8]. 
This research, utilizing the relatively complete sequence data of both genomic DNA and cDNA of Arabidopsis, aims to explore the correlation between the non-coding (both 5' and 3') and coding sequence regions. The situation at genome DNA level with inserted length of introns was also considered. Correlations between intron size and genome size among diverse range of organisms have been found and the presence of introns presents an interesting case [9]. Since genes in eukaryotes are located on different chromosomes and their products can be classified based on protein functions, we have not only analyzed the sequence length correlation at whole species level but also investigated the potential variations between different chromosomes and protein functional groups.

\section{Materials and Methods}

\subsection{Quantitative Measurements}

The gene length can be divided into three sections, and data was collected for each region with or without introns. As shown in Figure 1, the first region is situated between the Translation Start Site (TLS) and the translation stop codon (TSC). This region will be referred to as $D_{l}$, or coding region length (TLS-TSC distance) for the rest of this paper. The second region encompasses the +1 position after the promoter (the Transcription Start Site (TSS)) to the last nucleotide before the TLS. This region will be referred to as $D_{2}$ (TSS-TLS distance). The third and final region lies between the translation stop codon (TSC) and the transcription termination site (TTS), and will be referred to as $D_{3}$ (TSC-TTS distance). The distances were measured in base pairs (bp) of the nucleotide sequence. The data collected without introns will be denoted as $d_{1}, d_{2}$ and $d_{3}$ [10].

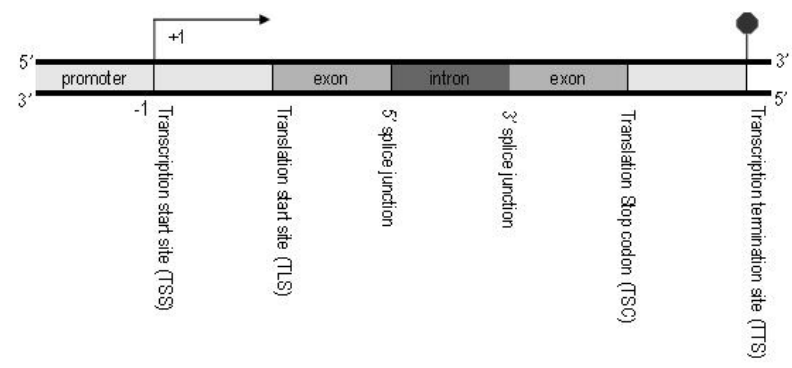

Figure 1. Diagrammatic illustration of a structural gene [10]

\subsection{Data Source and Processing}

The NCBI reference sequence assembly database, which was provided by The Arabidopsis Information Resource (TAIR) provided a comprehensive table on the coding sequence data [http://www.ncbi.nlm.nih.gov/sites/entrez?db=genom eprj\&cmd $=$ Retrieve \&dopt $=$ Overview\&list_uids $=116$ ]. The coding sequence (CDS) length was calculated from the start and stop position reference from the genome. The coding sequence lengths that did not contain introns were calculated from the protein length values.

Un-translated regions ( $3^{\prime}$ and 5' ends) were collected (March 2008) from the TAIR website [http://www.arabidopsis.org/help/helppages/BLAST_ help.jsp\#datasets].

Function classification of proteins was obtained from the COG (Clusters of Orthologous Groups of proteins) database [http://www.ncbi.nlm.nih.gov/COG/]. The data tables were combined into one table using Microsoft Access (2003), matching the locus tag ID. All three regions of data were combined into a master table which included values with and without introns and separated into chromosomes.

\subsection{Statistical Analysis}

Comparisons were conducted on the ratio of each region (length value over total). In the following study, we denote $D_{i}^{*}=D_{i} /\left(D_{1}+D_{2}+D_{3}\right)$ and $d_{i}^{*}=d_{i} /\left(d_{1}+d_{2}+d_{3}\right), \quad i=1,2,3$. After initial statistical tests, it was found there was significant correlation between $D_{1}^{*}$ and $D_{3}^{*}\left(d_{1}^{*}\right.$ and $d_{3}^{*}$ ) values conducted in JMP software. However, closer analysis found there was a significant nonlinear relationship between the coding region length $d_{1}\left(D_{1}\right)$ and the non-coding region length $d_{2}^{*}\left(D_{2}^{*}\right)$.

Since the correlation between the coding region and non-coding regions is conditional on $d_{2}^{*}\left(D_{2}^{*}\right)$, these values were used to examine whether there may be some relationship between the $d_{2}^{*}\left(D_{2}^{*}\right)$ values and the protein functional groups. The data was separated into eight subsets based on data mining research techniques. 


\section{Results and Discussion}

\subsection{Length Distributions among all Three Regions}

Data from several databases were combined to include the length distances in base pairs (bp) for each region of interest, including the two untranslated regions and the coding region. The values either included introns $(D)$ or excluded introns $(d)$. The median values for the whole organism ranged from under $200 \mathrm{bps}$ for the 5' un-translated region to over $1600 \mathrm{bps}$ for the coding sequence. The 3' untranslated region values were just over $200 \mathrm{bps}$ (Figure 2). These values are comparable with previous interpretations on this organism [7]. Other studies have shown that the 5' UTR average lengths range between 100 and 200 nucleotides. The 3' UTR for plants range from about 200 nucleotides [8].

The data was split to chromosome level, and the median values were collected for each region. Little variation was observed between chromosomes, in each of the region categories (Figure 3).

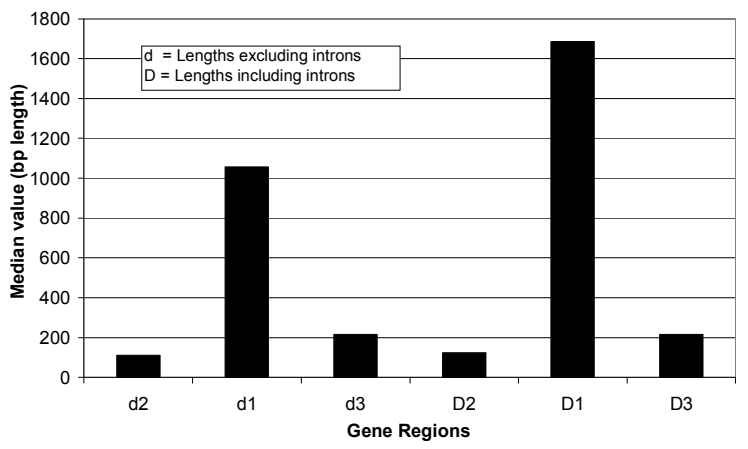

Figure 2. Median values of length distributions of Arabidopsis for the 5' UTR, coding sequence and 3' UTR. Data was combined from several databases. The sample size for $D$ (including introns) values was $N=25,965$. The sample size for $d$ (excluding introns) values was $\mathrm{N}=\mathbf{2 0 , 6 6 0}$

The average length (bp) of the coding sequence without introns $(d)$ is $\sim 68 \%$ of that of the coding sequence with introns $(D)$. Percentages of UTRs containing introns were calculated by Mignone et al in 2002 from $15-35 \%$ for 5 ' UTRs to $2-11 \%$ for 3 ' UTRs [8]. This has been confirmed in a recent study investigating abundance, distribution and intron size within un-translated regions of genes. It was established that the occupancy of introns in 5' UTRs of Arabidopsis thaliana (2,012 numbers of introns) is lower than in the coding sequence $(55,510)$, and with the 3' UTR (382), it contained even smaller amounts of introns than that of the 5' UTR [11]. Our data presented in Figure 2 also reflects this feature. The coding sequence data shows considerable differences between the values with introns $(D)$, to those without introns $(d)$. However, in the UTR data, there seems to be little difference in each value. This could be explained by the deficiency of intron data for these regions.

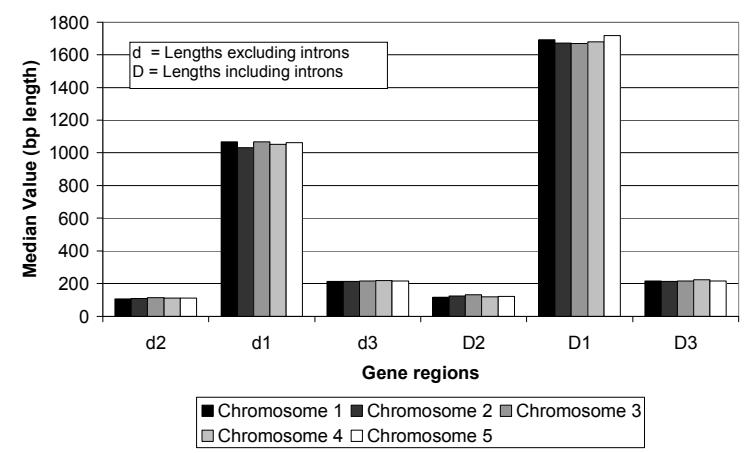

Figure 3. Median values of length distributions of Arabidopsis for the 5' UTR, coding sequence and 3' UTR segmented into chromosomes. Data was combined from several databases

\subsection{Nonlinear Function relationship between $D_{1}\left(d_{1}\right)$ and $D_{2}^{*}\left(d_{2}^{*}\right)$ Values}

Bivariate analysis was applied to the data for each region and chromosome. Through data mining techniques, a nonlinear function relationship can be established between $d_{1}$ and $d_{2}^{*}\left(D_{1}\right.$ and $\left.D_{2}^{*}\right)$ given the value of $\log \left(d_{2}\right)$ at different regions. The models used to fit the data are:

$$
\begin{aligned}
& d_{1}=\beta_{0}+\beta_{1} d_{2}^{*}+\beta_{2}\left(d_{2}^{*}\right)^{2}+e \\
& \text { and } \\
& D_{1}=\beta_{0}+\beta_{1} D_{2}^{*}+\beta_{2}\left(D_{2}^{*}\right)^{2}+e
\end{aligned}
$$

Figure 4 shows the scatter plot of $d_{1}$ verses $d_{2}^{*}$ for Chromosome 4 data. Figures 5-8 show the $\log \left(d_{2}\right)$ values split into six subsets for each chromosome, and the fitting $R^{2}$ values, as well as the estimations of parameters were plotted. The output for data with introns $(D)$, as well as the standard mean and square errors is excluded. 


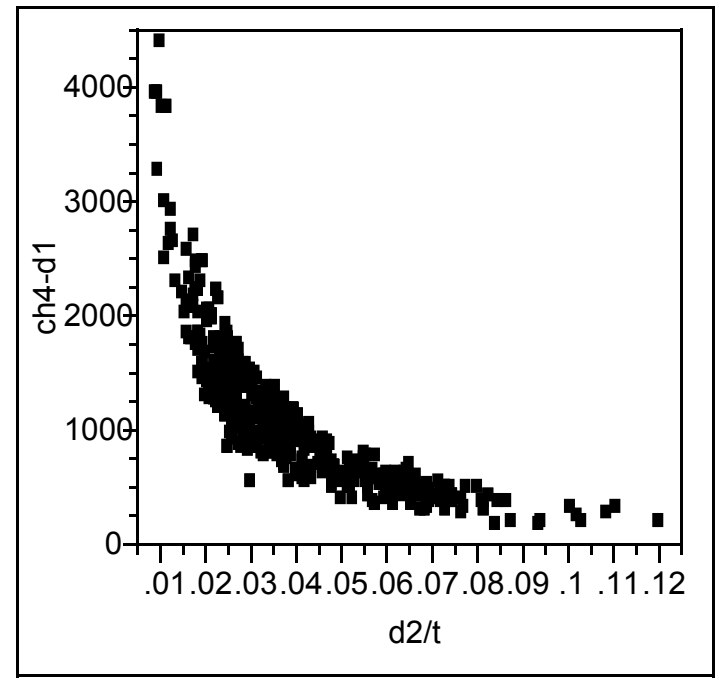

Figure 4. Scatter plot of $d_{1}$ and $d_{2}^{*}$ given by data of Chromosome 4 and $3.5 \leq \log \left(d_{2}\right)<4$

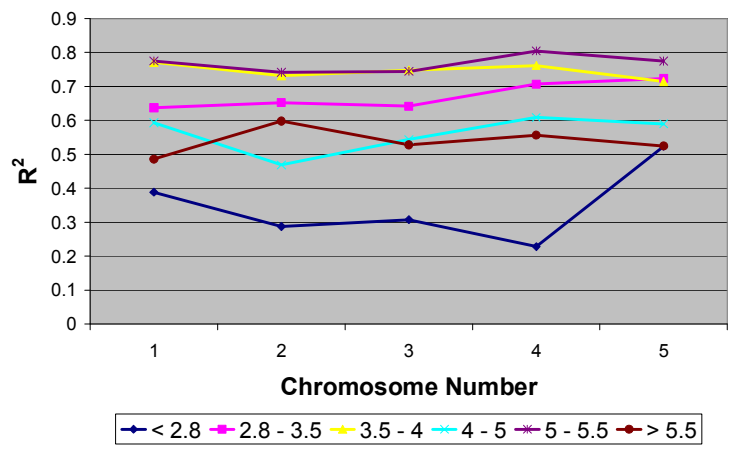

Figure 5. $R^{2}$ value's given by regression model for dataset not containing introns (d) over five chromosomes. Data was split into 6 subsets based on the $\log \left(d_{2}\right)$ values

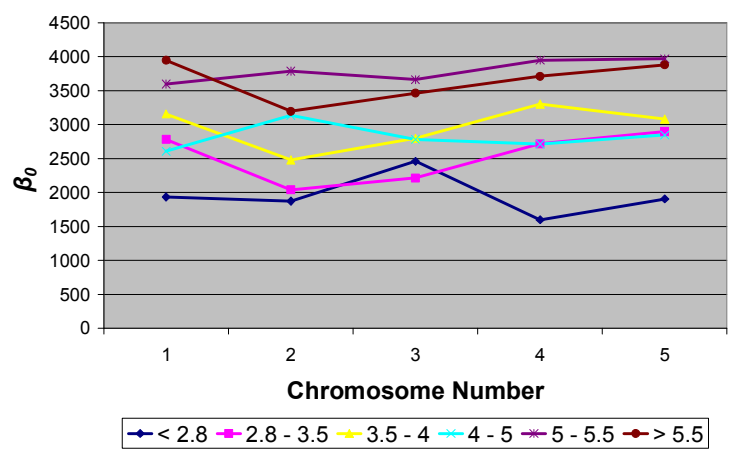

Figure 6 . The estimation of $\beta_{0}$ given by the data without introns (d) over five chromosomes. Data was split into 6 subsets based on the $\log \left(d_{2}\right)$ values

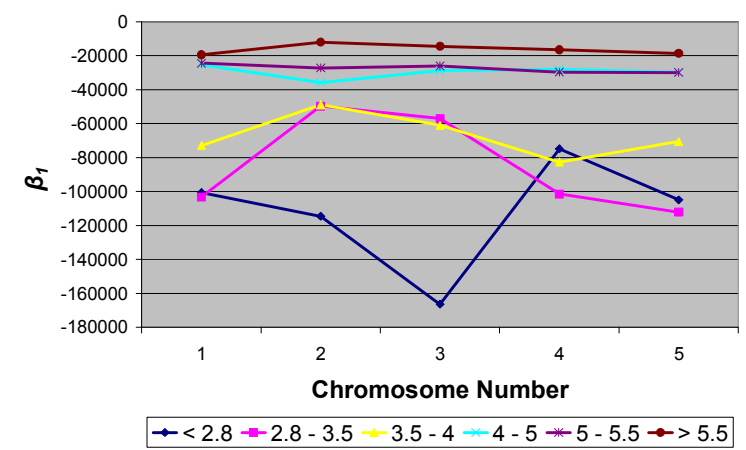

Figure 7. The estimation of $\beta_{1}$ given by the data without introns (d) over five chromosomes. Data was split into 6 subsets based on the $\log \left(d_{2}\right)$ values

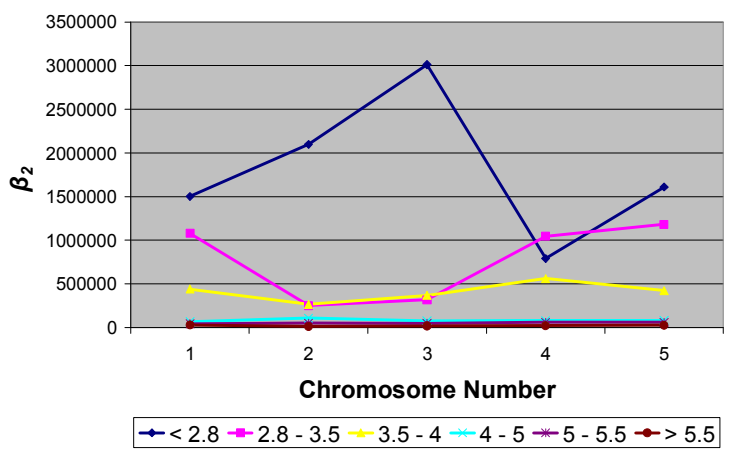

Figure 8. The estimation of $\beta_{2}$ given by the data without introns (d) over five chromosomes. Data was split into 6 subsets based on the $\log \left(d_{2}\right)$ values

Given the $\log \left(d_{2}\right)$ values of $<4$, the $R^{2}$ values given by the models are generally high. However, as $\log \left(d_{2}\right)$ values increase from 4 , the $R^{2}$ value tend to decrease and has greater variance across the chromosomes. From Figures 6-8, it was found that the models are varied based on which subset the $\log \left(d_{2}\right)$ belonged to. In general, as the value of the $\log \left(d_{2}\right)$ increases, the value of $\beta_{2}$ decreased, whereas $\beta_{0}$ and $\beta_{1}$ increased. However, when the $\log \left(d_{2}\right)$ value was $<2.8$, variability was seen in the estimations of $\beta_{0}, \beta_{1}$, and $\beta_{2}$ across all chromosomes, which could be explained by the small size of sample. In this preliminary study, we did not focus on 
the issue of how to classify the values of $\log \left(d_{2}\right)$ and further analysis on the data may provide an enhanced model for fitting the data. Future research is required to incorporate many more organisms in this type of research to confirm this nonlinear trend.

It is very interesting to note this nonlinear function relation between 5' UTR and the coding sequence, as well as the 3' UTR regions. Its significance in gene regulation and evolution warrants further investigation. This nonlinear relationship could be applied to prediction of length distributions of the 5' UTR region. If the coding sequence and the 3' UTR sequence length are known, the 5' UTR length could be predicted, which could provide guidance in promoter studies $[13,14]$. The values of $\log \left(d_{2}\right)$ has a big impact on model fitting as well as the confidence on the prediction of the value of $d_{2}\left(D_{2}\right)$. Inappropriately grouping the values of $\log \left(d_{2}\right)$ might limit this application.

\subsection{Differences between Protein Functional Classes}

Constraints on the evolution of proteins may be influenced by specific function, such as enzymes, regulators or signaling molecules [12]. Examination of protein lengths in conjunction with functional classes, such as cellular processes and metabolism identified that the protein lengths for these functions were greater than those of some other types. To investigate the sequence length distributions in different protein functional groups, the Arabidopsis sequence length data was ranked into four main categories based on basic functional classification (Table 1).

Table 1. COG Functional Classification [http://www.ncbi.nlm.nih.gov/COG/]

\begin{tabular}{ll}
\multicolumn{2}{l}{ Information storage and processing } \\
$\mathrm{J}$ & Translation, ribosomal structure and biogenesis \\
$\mathrm{A}$ & RNA processing and modification \\
$\mathrm{K}$ & Transcription \\
$\mathrm{L}$ & Replication, recombination and repair \\
$\mathrm{B}$ & Chromatin structure and dynamics \\
Cellular processes and signaling \\
$\mathrm{D}$ & Cell cycle control, cell division, chromosome partitioning \\
$\mathrm{Y}$ & Nuclear structure \\
$\mathrm{V}$ & Defense mechanisms \\
$\mathrm{T}$ & Signal transduction mechanisms \\
$\mathrm{M}$ & Cell wall/membrane/envelope biogenesis \\
$\mathrm{N}$ & Cell motility \\
$\mathrm{Z}$ & Cytoskeleton \\
$\mathrm{W}$ & Extracellular structures \\
$\mathrm{U}$ & Intracellular trafficking, secretion, and vesicular transport \\
$\mathrm{O}$ & Posttranslational modification, protein turnover,
\end{tabular}

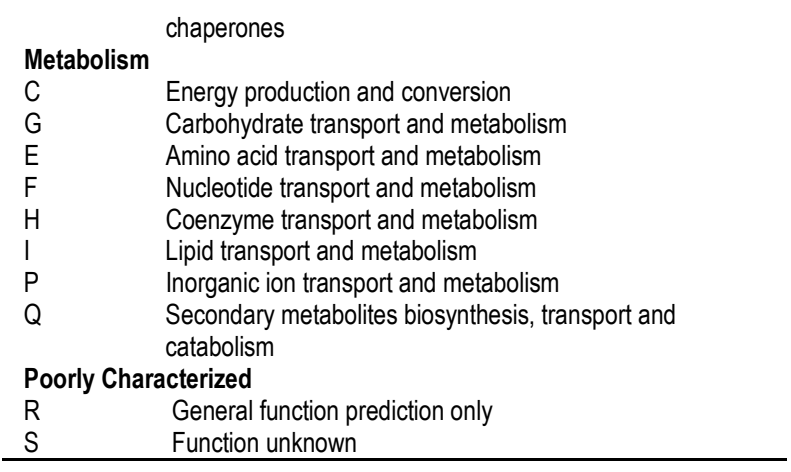

It was observed that as the $D_{2}^{*}\left(d_{2}^{*}\right)$ values in each of the eight subsets (start to $<0.05 ; 0.05$ to $<$ $0.1 ; 0.1$ to $<0.15 ; 0.15$ to $<0.2 ; 0.2$ to $<0.25 ; 0.25$ to $<0.3 ; 0.3$ to $<0.4$; and 0.4 to end), ascended from the smallest to the largest value, the number of different types of proteins in specific functional categories decreased. Investigation of chromosome 1 without introns showed that the different types of proteins in the cellular processes and signaling classification decreased as the $d_{2}^{*}$ values increased. However the other values for the other three categories were consistent over all subsets, showing little change. This was also seen in chromosome 2 and 4 (data not shown).

A similar trend was also seen in the metabolism functional class, again as the $D_{2}^{*}\left(d_{2}^{*}\right)$ values in each subset increased the number of different types of proteins decreased. Interestingly, this trend was only observed in chromosome 2 and 3 (data not shown).

Chromosome 4 and 5 were the only chromosomes that contained the nuclear structure protein type, and was only exposed in the smaller $D_{2}^{*}\left(d_{2}^{*}\right)$ values. Chromosome 5 of the Arabidopsis thaliana contains very similar genes to other organisms, including the human. This similarity can be propitious not only in the study of crop improvement but in health studies, as well as eukaryote comparison studies [15].

\section{Conclusion}

Our work has shown that there are possible correlations between the 3' and 5' UTR's and coding sequence lengths, although the data obtained in this paper was only based on Arabidopsis. Once more data is complied and analyzed, a larger picture of how these regions correlate should appear. In delving into the patterns of statistical properties of different gene regions and their correlation it is intended to elucidate the spatial organization rules between various gene functional elements and the 
difference in such organizations among different living organisms and gene families. It is assumed that these rules and differences are the results of evolution and reflect the complexity differences in the regulation of gene expression.

\section{References}

[1] J. Zhang, "Protein-length distributions for the three domains of life", Trends in Genetics, 16, 2000, pp. 107109 .

[2] L. Brocchieri, and S. Karlin, "Protein length in eukaryotic and prokaryotic proteomes", Nucleic Acids Research, 33, 2005, pp.3390-3400.

[3] T. Tan, D. Frenkel, V. Gupta, and M. W. Deem, "Length, protein-protein interactions, and complexity, Physica A, 350, 2005, pp. 52-62.

[4] F. Chiaromonte, W. Miller, and E. E. Bouhassira, "Gene length and proximity to neighbours affect genomewide expression levels", Genome Research, 13, 2003, pp. 2602-2608.

[5] Y. Dai, R. Zhang, and Y.-X Lin, "The probability Distribution of distance TSS-TLS is organism characteristic and can be used for promoter prediction, In: Ali M And Daposigny $\mathrm{R}$ (eds) Advances in Applied Artificial Intelligence - Lecture Notes in Artificial Intelligence (LNAI 4031), Springer, pp. 927-934.

[6] The Arabidopsis Genome Initiative, "Analysis of the genome sequence of the flowering plant Arabidopsis thaliana", Nature, 408, 2000, pp.796-815.

[7] N. N. Alexandrov, M E. Troukhan, V V. Brover, T. Tatarinova, R. B. Flavell and K. A. Feldmann, "Features of Arabidopsis genes and genome discovered using full-length cDNAs", Plant Molecular Biology, 60, 2006, pp. 69-85.

[8] F. Mignone, C. Gissi, S. Liuni, and G. Pesole, "Untranslated regions of mRNAs", Genome Biology, 3, 2002, pp. 0004.1-0004.10.

[9] A. E. Vinogradov, "Growth and decline of introns", Trends in Genetics, 18(5), May 2002, pp. 232-236.

[10] R. Caldwell, Y. Dai, S. Srivastava, Y. Lin, and R. Zhang, "Improving neural network promoter prediction by exploiting the lengths of coding and non-coding sequences", Chapter: Advances of Computational intelligence in industrial systems (Studies in Computational intelligence) edited by Ying Liu, Aixin Sun, Han Tong Loh, Wen Feng Lu and Ee-Peng Lim, Springer, 2008, pp. 213230.

[11] X Hong, D. G. Scofield, and M. Lynch, "Intron Size, Abundance, and Distribution within Untranslated regions of Genes", Molecular Biology Evolution, 23, 2006, pp. 2392-2404.

[12] D. J. Lipman, A. Souvorov, E. V. Koonin, A. R. Panchenko, and A. Tatusova, "The relationship of protein conservation and sequence length", BMC Evolutionary Biology, 2, 2002, pp. 1-10.

[13] S. Burden, Y. Lin, and R. Zhang, "Improving promoter prediction for the NNPP2.2 algorithm: a case study using Escherichia coli DNA sequences", Bioinformatics, 21, 2005, pp. 601-607.

[14] V. B. Bajic, S. L. Tan, Y. Suzuki, and S. Sugano, "Promoter prediction analysis on the whole human genome", Nature Biotechnology, 22, 2004, pp. 1467-1473

[15] The Kazusa DNA Research Institute, The Cold Spring Harbor and Washington University in St Louis Sequencing Consortium \& The European Union Arabidopsis Genome Sequencing Consortium, "Sequence and analysis of chromosome 5 of the plant Arabidopsis thaliana", Nature, 408(14), 2000, pp. 823-826. 